\title{
Trustworthy objects
}

\author{
Elisa Bertino
}

DSI - Universita di Milano

Via Comelico, 39/41, I-20135 Milano

bertino@dsi.unimi.it

ABSTRACT. As all modern organizations increasingly depend on computerized systems and applications, we need to build systems that can be trusted. Even though trust can be defined in many different ways, we can say that trusted systems have three primary characteristics: (a) authenticity - users of a computer system are confident that they know to whom and which entity they are interacting with; (b) integrity - users must have assurance that the information is transmitted accurately; (c) confidentiality and privacy - users must have assurance that the confidentiality of information as well of their personal data is respected. Building systems able to meet all those requirements is still a difficult task that requires efforts and research from different areas of computer science, from formal foundations, to programming languages and methodologies, to data management systems, just to name a few. In the talk, we discuss the above aspects of trust and present an overview of work done in secure object systems - thus addressing some relevant trust aspects, with special emphasis on object data management systems. We then outline open research directions.

RÉSUMÉ. Du fait que les organisations modernes sont de plus en plus dépendantes des systèmes et applications informatiques, il nous faut pouvoir construire des systèmes dignes de confiance. Bien que la confiance puisse être définie de plusieurs manières différentes, nous dirons que des systèmes dignes de confiance ont trois caractéristiques principales : (a) authenticité - les utilisateurs d'un système informatique sont sûrs de savoir avec qui et avec quelle entité ils interagissent; (b) intégrité - les utilisateurs doivent avoir l'assurance que l'information est transmise de manière exacte ; (c) confidentialité - les utilisateurs doivent avoir l'assurance que la confidentialité de l'information ainsi que de leurs données personnelles est respectée. La construction de systèmes capables de garantir toutes ces propriétés est une tâche encore difficile qui nécessite des efforts et des recherches provenant de différents secteurs de l'informatique : des fondations formelles, aux langages et méthodologies de programmation, jusqu'aux systèmes de gestion de bases de données, pour en citer quelques-uns. Dans cet exposé, nous discutons de ces problèmes de confiance et nous présentons un survol des travaux dans le domaine de la sécurité des systèmes à objets. Nous traitons ainsi certains aspects significatifs en matière de confiance, notamment pour les systèmes de gestion de bases de données à objets. Enfin, nous présentons des directions de recherche ouvertes.

KEYWORDS: security, object data management systems, trust management.

MOTS-CLÉS : sécurité, systèmes de gestion de bases de données à objets, gestion de la confiance. 